\title{
All plugged up - noninvasive mucus score to assess airway dysfunction in asthma
}

\author{
Steve N. Georas \\ Division of Pulmonary and Critical Care Medicine, University of Rochester Medical Center, Rochester, New York, USA.
}

\begin{abstract}
Asthma is remarkably heterogeneous, and there are multiple underlying inflammatory pathways and structural airway abnormalities that lead to symptomatic disease. Consequently, a current challenge in the field is to precisely characterize different types of asthma, with the goal of developing personalized approaches to therapy. In the current issue of the $J C I$, Dunican et al. developed a noninvasive way to assess airway dysfunction in asthma by measuring mucus accumulation using multidetector computed tomography (MDCT) and found that mucus plugging of small airways was remarkably common in subjects with severe asthma. This work highlights the importance of noninvasive imaging approaches in defining specific asthma subsets and guiding targeted therapies.
\end{abstract}

The corresponding mucus score (ranging from 0 to 20) had good interobserver variability and detected mucus plugs that were evenly distributed in all five lung lobes and were remarkably stable over time. Mucus plugs were detected in at least one lung segment in $58 \%$ of asthmatics compared with only one of twenty-two control subjects (4.5\%). The asthma subjects in this study were part of the NIH-sponsored Severe Asthma Research Program (SARP), which enrolled distinct groups of patients. High mucus scores (defined as $>4$ lung segments involved) were detected in between $44 \%$ and $64 \%$ of the SARP cohorts examined.

\section{Mucus score to determine airflow obstruction}

Asthma is a common disease characterized by intermittent wheezing, chest tightness, and cough and is caused by airway inflammation and airflow obstruction. Overproduction of mucus is thought to contribute to asthma pathophysiology, but this is difficult to measure in individual patients. Because it traps inhaled pathogens and particles, airway mucus is an essential part of epithelial host defense mechanisms (1). Mucus is a mixture of water, glycosylated mucins, and other nonmucin components and has the consistency of a thin gel that is propelled upward by the beating of cilia and is cleared out of the lungs and swallowed. However, mucus can become thick and tenaciously adhere to airway walls (Figure 1) in airway diseases. Although tenacious mucus is a nuisance for patients who note "difficulty clearing secretions," it can also be dangerous. First, mucus that is not propelled out of the lungs acts as a reservoir of inhaled pathogens and leads to persistent airway infections, as occurs in patients with cystic fibrosis (CF). Second, if developed in multiple large airways, mucus plugs can cause airflow obstruction. In fact, obstruction of large airways by tenacious mucus plugs is the principal cause of respiratory failure in patients who die suddenly of acute asthma attacks (2). Third, increasing evidence points to a key role for noncontractile airway narrowing due to mucus and mucus plugs in allergic airway hyperresponsiveness $(3,4)$. In this issue of the JCI, Dunican et al. report a new noninvasive way to measure airway mucus accumulation in asthma using multidetector computed tomography (MDCT) (5). They developed a reproducible "mucus score" and found that mucus plugging of small airways was remarkably common in subjects with severe asthma. Subjects with high mucus scores had more severe airflow obstruction and higher indices of eosinophilic airway inflammation. Consequently, the airway mucus score is a new noninvasive way to assess airway dysfunction in asthma that should be useful in future clinical research.

Dunican et al. developed an algorithm that distinguishes opacification of the airway lumen from adjacent blood vessels and quantified this in 20 lung segments.

Related Article: p. 997

Conflict of interest: The author has declared that no conflict of interest exists.

Reference information: J Clin Invest. 2018;128(3):906-909. https://doi.org/10.1172/JCI99726.

\section{Mucus score useful despite some limitations}

The fact that there was no widespread atelectasis downstream of the observed plugs suggests that either the plugs were not totally occlusive, allowing some airflow into these subsegments, or that downstream lung units were supplied by collateral ventilation. These possibilities are not mutually exclusive. Subjects with mucus plugs detected by MDCT might also have other partially occluded airways that are not easily detected radiographically. It is easy to envision how partial occlusion of the airway lumen by adherent mucus can contribute to airflow obstruction. Since airway resistance is inversely related to airway radius to the fourth power, any structural abnormality that narrows luminal diameter will greatly enhance airway resistance and also exaggerate the effects of smooth muscle constriction on flow limitation. Partially occluded airways are also more prone to collapse during forced exhalation, which helps explain the observed association between mucus scores and reduced forced vital capacity (FVC). Tenacious mucus and mucus plugs might also act like a one-way valve and allow airflow during inspiration, which would lead to air trapping. This was supported by the observation that subjects with a high mucus 


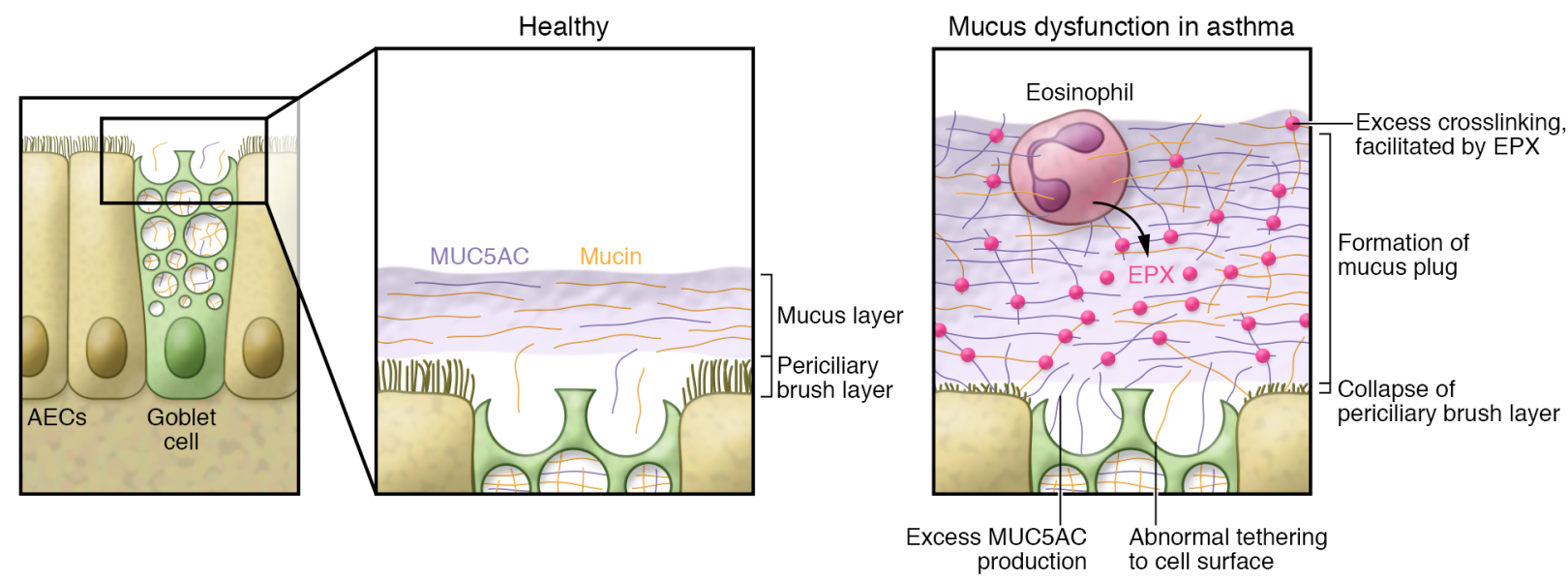

Figure 1. Mucus dysfunction in asthma. The airway epithelium made up of ciliated cells and goblet cells forming a tight barrier to the outside world. Inset: In a healthy airway epithelium, goblet cells secrete mucin proteins that are incorporated into a thick gel that coats the luminal side of airways and is propelled out of the lung by the beating of cell-surface cilia. In asthma and other airway disease, this mucociliary defense mechanism does not function as effectively and mucus can become trapped and form mucus plugs. Four general mechanisms of mucus dysfunction have been described, including the following: (a) mucus dehydration leading to collapse of the periciliary brush mucus layer, (b) excess production of mucins such as Muc5AC, (c) abnormal tethering of mucus strands to the goblet cell surface, and (d) mucus crosslinking during inflammation. In the presence of eosinophilic airway inflammation, eosinophil peroxidase (EPX), together with other oxidant molecules, can crosslink mucus strands, leading to a more viscous gel that is difficult to expel. These mechanisms likely contribute to the formation of mucus plugs in subjects with eosinophilic asthma. AEC, airway epithelial cell.

score had a higher ratio of residual volume (RV) to total lung capacity (TLC) on pulmonary function testing. A low FVC and elevated RV/TLC ratio is commonly observed in severe asthma (6) and has been attributed to excess bronchoconstriction or reduced lung elastic recoil $(7,8)$. The study by Dunican et al. suggests that narrowing of airways by tenacious mucus is another potential mechanism involved.

Subsegmental ventilation defects are also commonly observed in asthma (9) and, interestingly, were recently found to be associated with severe asthma exacerbations (10). It seems logical to speculate that mucus plugs detected by MDCT would cause ventilation defects observed using other imaging approaches; however, two observations suggest that this association may not be so straightforward. First, in the study by Zha et al., ventilation defects in asthma had a predilection for certain lung segments, such as right upper and middle lobes (11), as opposed to the uniform distribution of mucus plugs reported by Dunican et al. Second, high mucus scores were only weakly associated with asthma exacerbations in the study by Dunican et al. More research will be needed to determine exactly how mucus plugs detected by MDCT affect regional ventilation patterns in the lung and clinical phenotypes in asthma.
The human lung contains thousands of airways arranged in series and in parallel, and it may not be obvious as to how defects in ventilation to a few lung-segment units can have marked physiologic consequences. One potential explanation comes from the observation that airflow in the lung is nonlinear, and small differences in airway diameter can lead to large clusters of poorly ventilated lung units (12). In fact, ventilation heterogeneity as assessed using multiple breath nitrogen washouts is strongly associated with airway hyperresponsiveness in asthma (13). Additional research is warranted in order to study the relationship among airway occlusion by adherent mucus, ventilation heterogeneity, and airflow obstruction in asthma.

Although the study by Dunican et al. shows a clear association between high mucus scores and airflow obstruction, it is difficult to know whether this association is causal. That is, does excess mucus cause airflow obstruction, or does something else lead to the development of tenacious mucus plugs at sites of injured airways prone to obstruction? However, it is not possible to answer this question definitively in an observational study such as this. Future longitudinal studies might provide some insight into whether high mucus scores precede the development of airflow obstruction in at-risk subjects. More con- vincing evidence is expected to come from clinical trials designed to improve both mucus scores and airflow obstruction by targeted mucolytic therapies or therapies to reduce mucin overproduction or rapid secretion. Although no such therapies currently exist, this is an active area of research where new approaches will hopefully be available in the future.

\section{High mucus scores indicative of mucus dysfunction}

Mucus dysfunction is a hallmark of CF, but also occurs in chronic obstructive pulmonary disease (COPD) and asthma. The biochemical basis of mucus dysfunction in these diseases is complex, and multiple mechanisms are probably involved. Four general mechanisms of mucus dysfunction have recently been discovered (Figure 1). First, dehydration of airway mucus due to abnormal salt and water transport is thought to be a major cause of mucostasis in CF, leading to collapse of the periciliary brush layer and impaired mucociliary clearance (14). Second, the "gel-on-brush" model suggests that the relationship between secreted mucins and mucociliary transport is bell shaped, with paradoxically reduced mucus clearance after a critical threshold mucin concentration is reached (14). Therefore, excess production of gel-forming mucins includ- 
ing MUC5AC and MUC5B might actually lead to reduced mucus transport. Airway mucins are difficult to measure, but recent studies point to a role for mucin overproduction in both CF and COPD $(15,16)$. A third mechanism of mucus dysfunction in airway diseases that is potentially relevant to the high mucus scores observed by Dunican et al. is abnormal mucus tethering. This refers to the inability of newly synthesized mucus strands to detach from mucus-producing cells in the airway and was first described using novel imaging approaches in a pig model of CF (17). Interestingly, Bonser et al. recently reported a similar phenomenon in IL-13-stimulated human bronchial epithelial cells cultured in vitro (18). IL-13 is produced by type 2 $\mathrm{CD}^{+} \mathrm{T}$ cells (Th2) and innate lymphoid cells (ILC2s) and is a potent inducer of MU5AC production by airway goblet cells (19). Using immunofluorescence and imaging of microsphere transport with washed and unwashed epithelial monolayers, Dunican et al. concluded that IL-13 induces abnormal tethering of MUC5AC to the goblet cell surface, reducing mucociliary clearance. It is easy to imagine how abnormally tethered mucus could be the nidus for the mucus plugs detected by Bonser et al. In support of this possibility, high mucus scores were associated with elevated MUC5AC/MUC5B ratios in severe asthmatics, pointing to a pathologic role for MUC5AC.

Besides mucus dehydration, excess mucin production, or abnormal tethering, a fourth and emerging mechanism of mucus dysfunction in airway diseases relates to posttranslational modification of mucus polymers. For example, oxidation of mucus cysteines was recently shown to cause mucin crosslinking, leading to stiffer mucus gels (20). Dunican et al. propose that eosinophils cause a similar oxidant-dependent mechanism of mucus dysfunction in asthma. This proposition is supported by in vitro studies showing that eosinophil peroxidase (EPX), together with hydrogen peroxide and potassium thyocyanate, increased the elastic modulus of a model hydrogel. In the SARP asthma cohorts, high mucus scores were associated with sputum eosinophilia and EPX levels, supporting the association between eosinophils and mucus dysfunction. The discovery of oxidant-dependent mecha- nism of mucus dysfunction is exciting and opens the door to targeted therapeutics that might reduce mucus viscosity. Additionally, it will be interesting to see if targeted antieosinophil strategies reduce mucus scores in severe asthmatics. A challenge in the development of mucus-targeted drugs is that airway mucins are an essential part of the host defense mechanism; therefore, strategies that broadly inhibit mucus production might not be well tolerated, although reduction of MUC5AC with preservation of MUC5B could be a viable strategy (21). It is also clear that secreted and membrane-tethered mucins are not just inert gels and can regulate airway inflammation and immunity (22-24). Therefore, future clinical trials of compounds targeting mucus production will need to consider possible unexpected effects on airway immune responses.

\section{Summary}

In summary, the development of the radiographic mucus score by Dunican et al. represents an elegant example of translational research that will further our understanding of asthma heterogeneity. This is an exciting time to be doing asthma research, since advances in our understanding of asthma pathophysiology are being translated into personalized approaches to diagnosis and treatment. Although most current research is aimed at uncovering specific inflammatory pathways in asthma, this study reminds us that we need to consider airway structural defects as well. Perhaps in the not too distant future, noninvasive imaging approaches will help us define specific asthma subsets and guide targeted therapies.

\section{Acknowledgments}

This work was supported by NIH/NHLBI R01HL12242. I thank Wayne Mitzner and Burton Dickey for helpful discussions.

Address correspondence to: Steve N. Georas, Division of Pulmonary and Critical Care Medicine, University of Rochester Medical Center, Rochester, New York 14642, USA. Phone: 585.275.4861; Email: Steve_Georas@urmc.rochester.edu.

1. Fahy JV, Dickey BF. Airway mucus function and dysfunction. $N$ Engl J Med. 2010;363(23):2233-2247.
2. Kuyper LM, et al. Characterization of airway plugging in fatal asthma. Am JMed. 2003;115(1):6-11.

3. Bossé Y, Riesenfeld EP, Paré PD, Irvin CG. It's not all smooth muscle: non-smooth-muscle elements in control of resistance to airflow. Annu Rev Physiol. 2010;72:437-462.

4. Evans CM, et al. The polymeric mucin Muc5ac is required for allergic airway hyperreactivity. Nat Commun. 2015;6:6281.

5. Dunican EM, et al. Mucus plugs in patients with asthma linked to eosinophilia and airflow obstruction. J Clin Invest. 2018;128(3):997-1009.

6. Sorkness RL, et al. Lung function in adults with stable but severe asthma: air trapping and incomplete reversal of obstruction with bronchodilation. JAppl Physiol. 2008;104(2):394-403.

7. Gibbons WJ, Sharma A, Lougheed D, Macklem PT. Detection of excessive bronchoconstriction in asthma. Am J Respir Crit Care Med. 1996;153(2):582-589.

8. Gelb AF, et al. Risk factors for near-fatal asthma. Chest. 2004;126(4):1138-1146.

9. Teague WG, Tustison NJ, Altes TA. Ventilation heterogeneity in asthma. J Asthma. 2014;51(7):677-684.

10. Mummy DG, et al. Ventilation defect percent in helium-3 magnetic resonance imaging as a biomarker of severe outcomes in asthma [published online ahead of print November 10, 2017]. JAllergy Clin Immunol. https://doi.org/10.1016/ j.jaci.2017.10.016.

11. Zha W, et al. Regional heterogeneity of lobar ventilation in asthma using hyperpolarized helium-3 MRI [published online ahead of print November 23, 2017]. Acad Radiol. https://doi. org/10.1016/j.acra.2017.09.014.

12. Venegas JG, et al. Self-organized patchiness in asthma as a prelude to catastrophic shifts. Nature. 2005;434(7034):777-782.

13. Downie SR, Salome CM, Verbanck S, Thompson B, Berend N, King GG. Ventilation heterogeneity is a major determinant of airway hyperresponsiveness in asthma, independent of airway inflammation. Thorax. 2007;62(8):684-689.

14. Button B, et al. A periciliary brush promotes the lung health by separating the mucus layer from airway epithelia. Science. 2012; 337(6097):937-941.

15. Henderson AG, et al. Cystic fibrosis airway secretions exhibit mucin hyperconcentration and increased osmotic pressure. J Clin Invest. 2014;124(7):3047-3060.

16. Kesimer M, et al. Airway mucin concentration as a marker of chronic bronchitis. $N$ Engl J Med. 2017;377(10):911-922.

17. Hoegger MJ, et al. Impaired mucus detachment disrupts mucociliary transport in a piglet model of cystic fibrosis. Science. 2014;345(6198):818-822.

18. Bonser LR, Zlock L, Finkbeiner W, Erle DJ. Epithelial tethering of MUC5AC-rich mucus impairs mucociliary transport in asthma. JClin Invest. 2016;126(6):2367-2371.

19. Kuperman DA, et al. Direct effects of interleukin-13 on epithelial cells cause airway hyperreactivity and mucus overproduction in asthma. Nat Med. 2002;8(8):885-889.

20. Yuan S, et al. Oxidation increases mucin polymer 
cross-links to stiffen airway mucus gels. Sci Transl Med. 2015;7(276):276ra27.

21. Roy MG, et al. Muc 5 b is required for airway defence. Nature. 2014;505(7483):412-416.

22. Kim KC, Lillehoj EP. MUC1 mucin: a peace- maker in the lung. Am J Respir Cell Mol Biol. 2008;39(6):644-647.

23. Shan M, et al. Mucus enhances gut homeostasis and oral tolerance by delivering immunoregulatory signals. Science. 2013;342(6157):447-453.
24. Janssen WJ, Stefanski AL, Bochner BS, Evans CM. Control of lung defence by mucins and macrophages: ancient defence mechanisms with modern functions. Eur Respir J. 2016;48(4):1201-1214 